\title{
Kinematic analysis, modeling and simulation of the substation inspection robot
}

\author{
Jinbo Zhang ${ }^{1,2, a}$, Zong $\mathrm{Wu}^{1, \mathrm{~b}}$, Shuai $\mathrm{Lu}^{1, \mathrm{c}}$ \\ $1 \mathrm{HoHai}$ University College of Internet of Things Engineering, Changzhou, Jiangsu 213022,China \\ 2 Key Laboratory of intelligent technology and special robot, Changzhou, Jiangsu 213022,China \\ ajbzhang@hhu.edu.cn, bwuzong_0311124@163.com, 'lush1991@126.com
}

\begin{abstract}
Keywords: Transformer substation, Inspection robot, Kinematics, Simulation
Abstract: As a development direction of power grid, Smart grid has entered the stage of comprehensive construction, the detection and management of the intelligent substation's integrated parameter has become an increasingly prominent. The traditional manual inspection can't meet the requirements of modern intelligent substation and it is a trend that the substation inspection robot will replace the traditional manual inspection gradually. This paper is based on the kinematics model of the robot and drive motor's dynamics model. We use the Matlab/Simulink software to establish the mathematical model of mobile robot and conduct the model simulation according to the relevant parameters. The result of our research shows that the double closed-loop control which we adopt in this passage has a strong tracking ability and a strong anti-interference capability; it can completely meet the performance requirements of substation inspection robot.
\end{abstract}

\section{Introduction}

Ever since the 21st century, with the innovation and breakthroughs of technology and theory of semiconductors, science and technology is continuously progressing. At the same time, the deepening and innovation of the reform of electric power system has become the inevitable trend. Our nation is giving a strong support to the development of power grid, the automation degree of power grid is keep rising, in order to reduce the waste of manpower and material resources the daily patrol of the equipments in transformer substation also gradually tend to be less human or even unmanned patrol. The correct operation of power equipments in the substation is linked to the safety of production and operation in the substation. Thus, we should keep regular daily check of the electrical equipments; we should inspect, sample and estimate the operating parameters of the equipments to find out whether they are operating correctly. However, there are some problems of the traditional inspect methods, such as the difference of ability and working attitude and heavy work of the staffs working in the power station, which lead to lots of potential problems exist in the daily inspection and maintenance work of the Substation equipment and make the equipments can't operate correctly. Considering the actual problems in substation and potential problems in inspection and management mode, combining with the development of the current machine technology, we should reduce these potential problems by introducing the robots to substation and replace the traditional mode of inspection.

Usually, the inspection robots work in the outdoor substations, to some degree replaces the inspectors or totally replaces the inspectors to complete the inspection task. As a kind of mobile robot, using relevance navigation method (such as image processing and recognition, GPS navigation and inertial navigation) the inspection robots can fulfill the real time monitoring and inspection of the equipments in substation even if there is no staff inspecting the equipments. The inspection robots can improve the stability and reliability of the substation equipment and realize the automation of substation inspection. Thus this technique has a broad development prospect.

The inspection robot is a self-control system and the system control target of it is speed control and moving position. The control system is based on the principle of automatic control and the core of the system is the high-performance micro controller. The system fulfills the control task with the help of an actuator, which is formed by power converter consists of power electronic devices. Motion control accuracy determines the performance of the inspection robot and the self localization technology is the most important technology that we will study. Only when the robot has a certain degree of 
positioning accuracy in the process of movement, can the robot be used in the equipment inspection work in the substation.

\section{Analysis of the principle of differential steering of the inspection robot}

The inspection robot generally use four wheeled machinery structure, the two wheels in the front of the robot are power wheels powered by brushless DC motor, the other two wheels are driven wheel and are used to support the robot. We can know from the dynamics principle that if we want the robot to move in a given trajectory we have to control the speed of the two driving wheels precisely. Usually we can let the robot turn to another side by set a difference speed between the two power wheels. The mobile robot is a typical nonholonomic system. Due to the constraints of mechanical structure and external environmental constraints, the modeling of the nonholonomic system is difficult. Thus we have following four hypotheses when modeling and studying our topic.

(1) The robot moves in the two-dimensional plane and it is seen as point-contact rigid body.

(2) There is no longitudinal slip between the wheels and the ground when the robot moves.

(3) The contact between power wheels and the ground is point-contact and ignore the thickness of the wheels.

(4) The geometric distribution of the four driving wheels is symmetrical and the turn round of the robot is based on the skid.

Thus, in this paper, we use the differential steering principle to control the movement direction of robot. We make kinematics analysis of the robot in the plane and the structure of the car is shown in PIC-1. The two driving wheels are the main study object. $O 1$ and $O 2$ stands for the center of the two power wheels, the distance between them is L, the center of $O 1$ and $O 2$ is $C$, which means the center of gravity of the car. $V 1, V 2$ and $V_{C}$ stands for the velocity of two power wheels and the center of gravity.

Form a geometric model for position and motion of the mobile robot on geodetic coordinate system $X O Y$. We can know from the schematic diagram of the structure in Fig. 1 that the coordinate of the gravity center $C$ is $(X, Y)$, it is also the position of the robot in the moving space. When we use a counter clockwise direction as a positive direction, the angle $\theta$ between $V_{C}$ and x-axis stands for the motion state of the robot, we use coordinate $(X, Y, \theta)$ to represent the position of the robot in the coordinate system.

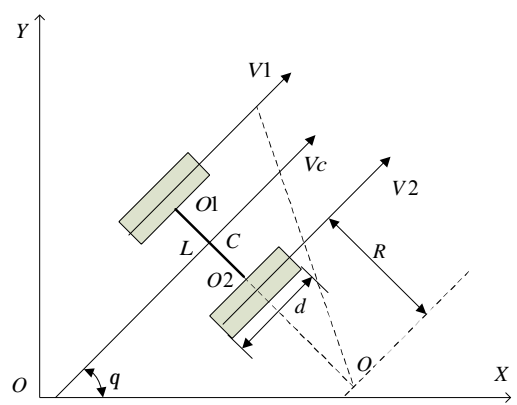

Fig.1- The schematic diagram of the structure of the robot

In Fig. 1, $O$ stands for the moving center of the robot. We assume that the contact between robot and ground is point-contact, the robot moves by roiling and the wheels don't sideslip. We assume the angular velocity of the robot body is $\omega$, when the robot moves in clockwise direction:

$$
V_{C}=\omega * R
$$

We can know from the rate decomposition formula that the velocity of the two wheels $V 1, V 2$ is:

$$
\left\{\begin{array}{l}
V 1=\omega *\left(R+\frac{L}{2}\right) \\
V 2=\omega *\left(R-\frac{L}{2}\right)
\end{array}\right.
$$


According to (1) and (2) giving above, when $V 1, V 2$ is already known, we can know the velocity of the gravity center of the $\operatorname{robot} C$ is:

$$
V_{C}=\frac{V 1+V 2}{2}
$$

The angular velocity of the robot body $\omega$ is:

$$
\omega=\frac{V 1-V 2}{L}
$$

According to (3) and (4) giving above, we can know the motion state of the robot powered by the difference between two wheels is determined by the rotation speed of two power wheels. We can let the mobile robot make rotary motion around a fixed point at a certain speed by controlling the speed of the two wheels of the robot.

The main task of the substation inspection robot is plan of inspecting the equipments in the substation along the set path. Thus we have to know the position of the robot in coordinate system $X O Y$ all the time during the control progress. If we have already known the position of the robot at time $^{t_{n}}$ and if $V 1, V 2$ is already known, after a while at time ${ }^{t_{n+1}}$ the change of position of robot is shown in Fig.2.

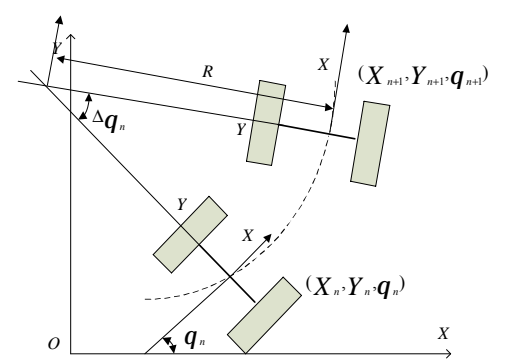

Fig.2-Change of position of the inspection robot

Fix the inspection robot body in coordinate system $X O Y$ on the ground, the gravity center of the robot $C$ coincide with the origin of coordinates $O$. As shown in Fig.2, the starting point of the robot's position is $\left(X_{n}, Y_{n}, \theta_{n}\right)$, after moving for $\Delta t$, its position becomes $\left(X_{n+1}, Y_{n+1}, \theta_{n+1}\right)$. According to the hypothesis that the contact between car and ground is point-contact, the motion of the robot in $\Delta t$ is circular motion, the turning angle in the move is $\Delta \theta=\theta_{n+1}-\theta_{n}, R$ in Fig.2is the trajectory radius of the robot's movement. We can infer the motion state $\left(X_{n+1}, Y_{n+1}, \theta_{n+1}\right)$ according to the motion state $\left(X_{n}, Y_{n}, \theta_{n}\right)$ :

$$
\left\{\begin{array}{l}
\Delta X_{n}=\Delta x_{n} \cos \theta_{n}-\Delta y_{n} \sin \theta_{n} \\
\Delta Y_{n}=\Delta x_{n} \sin \theta_{n}+\Delta y_{n} \cos \theta_{n} \\
\Delta \theta_{n}=\omega * \Delta t
\end{array}\right.
$$

Due to formula that:

$$
\left\{\begin{array}{l}
X_{n+1}=\Delta X_{n}+X_{n} \\
Y_{n+1}=\Delta Y_{n}+Y_{n} \\
\boldsymbol{\theta}_{n+1}=\Delta \boldsymbol{\theta}_{n}+\boldsymbol{\theta}_{n}
\end{array}\right.
$$

Combine with (5), (6) we can know that:

1) when the robot is in linear motion:

$$
\left\{\begin{array}{l}
\Delta x_{n}=V c^{*} \Delta t \\
\Delta y_{n}=0
\end{array}\right.
$$

2) when the robot is in spin motion: 


$$
\left\{\begin{array}{l}
\Delta x_{n}=0 \\
\Delta y_{n}=0
\end{array}\right.
$$

3) when the robot is in circular motion:

$$
\left\{\begin{array}{l}
\Delta x_{n}=R^{*} \sin \Delta \theta \\
\Delta y_{n}=R-R^{*} \cos \Delta \theta_{n}
\end{array}\right.
$$

If $V_{x}, V_{y}$ represent for $\mathrm{X}$-axis and $\mathrm{Y}$-axis velocity of the robot in coordinate system $X O Y$, then:

$$
\left\{\begin{array}{l}
V_{x}=X^{\&}=V c * \cos \theta=\frac{(V 1+V 2) \cos \vartheta}{2} \\
V_{y}=Y \&=V c * \sin \theta=\frac{(V 1+V 2) \sin \vartheta}{2}
\end{array}\right.
$$

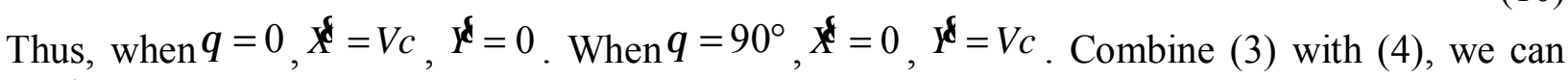
know that :

$$
R=\frac{(V 1+V 2) * L}{2(V 1-V 2)}
$$

According to the equations mentioned above, we can know the three motion states of the robots controlled by differential steering:

1) When $V 1=V 2: V c=V 1=V 2, R=+\infty$ and the robot is in linear motion.

2) When $V 1=-V 2: V c=0, R=0$ and the robot is in spin motion

3) When $V 1 \neq V 2: V c=(V 1+V 2) / 2$ and the robot is in circular motion, the moving radius is

$$
R=\frac{(V 1+V 2) L}{2(V 1-V 2)} \text {. }
$$

When can know from the thesis mentioned above that, no matter what kind of motion state the robot is, when $V$ and $\theta$ is given, we can control the linear velocity and angular velocity of the moving robot by controlling the speed of two wheels of the robot, we can also let the robot move in the giving orbit

\section{Modeling of the driving motor}

In the control system of the mobile robot, the driving motor is the power resource of the robot. the mathematical model describes the relationship between torque output to the power wheels and the electrical signal in the electric motor. Usually, we don't consider the model of the driving motor when we design and study the robots in order to design the control system easily. However, in this way, we don't take the high frequency interference into consideration, especially when the robot is used in a high speed and high accuracy condition. Thus, this kind of simplify is not reliable to a certain extent. In this paper, we will analysis the mathematical model of the motor when studying the motion robot.

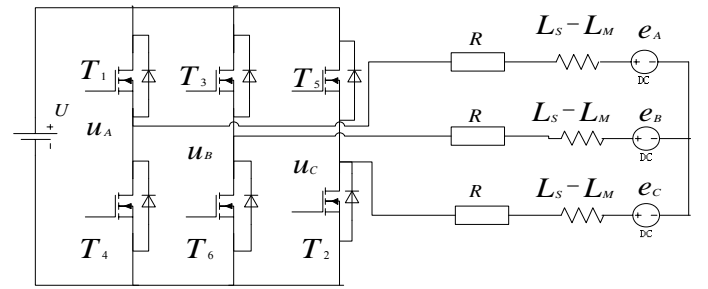

Fig.3- Equivalent circuit diagram of the brushless DC motor

We choose FBL-909H20301RGN20 as the brushless DC motor, this is a 24V DC motor, the number of magnetic pole is 4 , the rated current is $10.5 \mathrm{~A}$, the rated speed is $3000 \mathrm{RPM}$. The air gap magnetic field of brushless DC motor is non sinusoidal distribution.

Usually, the armature winding of brushless DC motor adopts star connection, no neutral point and it is three phase symmetrical. We also suppose that: 
1) We don't consider the magnetic saturation at motor core and we don't consider the hysteresis and eddy current losses.

2) The trapezoidal wave of the air gap magnetic is $120^{\circ}$ electric angle distributed.

3) We don't consider the cogging effect and the armature conductors distributed on the surface of armature uniformly.

4) The switch power tube and diode in the circuit have ideal switching characteristics.

We model and analyze the brushless DC motor based hypothesis on the mentioned above. According to the equivalent circuit diagram of the brushless DC motor, we can know that the three phase voltage equation of the brushless DC motor is:

$$
\left[\begin{array}{l}
u_{A} \\
u_{B} \\
u_{C}
\end{array}\right]=\left[\begin{array}{ccc}
R_{A} & 0 & 0 \\
0 & R_{B} & 0 \\
0 & 0 & R_{C}
\end{array}\right]\left[\begin{array}{l}
i_{A} \\
i_{B} \\
i_{C}
\end{array}\right]+\left[\begin{array}{ccc}
L_{A} & M_{A B} & M_{A C} \\
M_{B A} & L_{B} & M_{B C} \\
M_{C A} & M_{C B} & L_{C}
\end{array}\right] p\left[\begin{array}{l}
i_{A} \\
i_{B} \\
i_{C}
\end{array}\right]+\left[\begin{array}{c}
e_{A} \\
e_{B} \\
e_{C}
\end{array}\right]+\left[\begin{array}{l}
U_{n} \\
U_{n} \\
U_{n}
\end{array}\right]
$$

In the equation mentioned above, $L_{A}, L_{B}, L_{C}$ stand for the inductance of single phase winding; $e_{A}, e_{B}, e_{C}$ stand for the back electromotive force of each phase; $R_{A}, R_{B}, R_{C}$ stand for the equivalent resistance of each phase; $i_{A}, i_{B}, i_{C}$ stand for the value of current of each phase; ${ }^{M_{x y}}$ stand for the value mutual inductance between two phases(x and y stand for $\mathrm{A}, \mathrm{B}, \mathrm{C}$ phases); $P$ is the differential operator; ${ }^{U_{n}}$ stand for value of voltage at the neutral point of the motor. As the brushless DC motor is three phase symmetrical in the ideal case, $R_{A}=R_{B}=R_{C}=R, L_{A}=L_{B}=L_{C}=L, R_{A B}=R_{B C}=R_{C A}$. The connection type of the motor winding is Y. According to the KCL current theory, we can know that:

$$
i_{A}+i_{B}+i_{C}=0
$$

When put equation (13) into equation (12), we can achieve the following voltage balance equation:

$$
\left[\begin{array}{l}
u_{A} \\
u_{B} \\
u_{C}
\end{array}\right]=\left[\begin{array}{rrr}
R & 0 & 0 \\
0 & R & 0 \\
0 & 0 & R
\end{array}\right]\left[\begin{array}{c}
i_{A} \\
i_{B} \\
i_{C}
\end{array}\right]+\left[\begin{array}{ccc}
L-M & 0 & 0 \\
0 & L-M & 0 \\
0 & 0 & L-M
\end{array}\right] p\left[\begin{array}{l}
i_{A} \\
i_{B} \\
i_{C}
\end{array}\right]+\left[\begin{array}{l}
e_{A} \\
e_{B} \\
e_{C}
\end{array}\right]+\left[\begin{array}{l}
U_{n} \\
U_{n} \\
U_{n}
\end{array}\right]
$$

The output torque of motor stator is:

$$
T_{e}=\frac{P}{\omega}=\frac{e_{A} i_{A}+e_{B} i_{B}+e_{C} i_{C}}{\omega}
$$

In the equation mentioned above, ${ }^{T}$ stands for the output torque of motor stator; $P$ stands for the

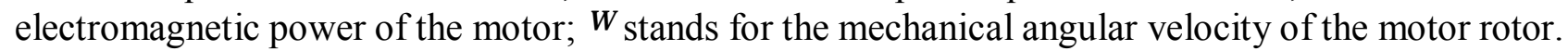
When the motor works correctly, the switch power tube of the inverter circuit unit in the motor breakover. Each time, only two phase windings are in conduction, the current in other winding is 0 , the current $i_{d}$ and the back electromotive force $E$ in the two conductive windings have the same value but opposite direction, the torque equation of the brushless DC motor can be simplified to:

$$
T_{e}=\frac{2 i_{d} E}{\omega}
$$

At the same time, the torque balance equation of the motor is:

$$
J \frac{d_{\omega}}{d_{t}}=T_{e}-T_{L}
$$

In the equation mentioned above, $J$ stands for the moment of inertia of rotor and load; $T_{L}$ stands for the torque of load. In the actual calculating, angular velocity $\omega$ is usually replaced with rotation rate $^{n}$; moment of inertia $J$ is usually replaced with the inertia of flywheel $G d^{2}$. Thus, we can get the following equations: 


$$
\begin{aligned}
& \omega=\frac{2 \pi n}{60} \\
& J=m \quad \rho^{2}=\frac{G d^{2}}{4 g}
\end{aligned}
$$

In these equations, $m$ stands for the mass of system's rotating part and its unit is $k g$; $G$ stands for

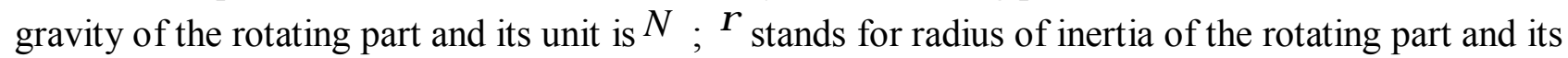
unit is $m$; $D$ stands for the radius of rotating inertia of system's rotating part and its unit is $m$; $g$ stands for acceleration of gravity. When put equations (18), (19) into equation (17), we can get the equation:

$$
T_{e}-T_{L}=\frac{G d^{2}}{375} \frac{d_{n}}{d_{i}}
$$

The dynamic voltage equation of the brushless DC motor can be expressed as:

$$
\begin{aligned}
& U=E+R i_{d}+(L-M) \frac{d_{i_{d}}}{d_{t}} \\
& \frac{d_{i_{d}}}{d_{t}}=\frac{U-E}{L-M}-\frac{i_{d}}{T_{l}}
\end{aligned}
$$

In these equations, $U$ stands for the DC voltage of inverter circuit and single phase winding; $E$ stands for back electromotive force in the single phase; ${ }^{T}$ stands for the constant of electromagnetic time in the motor; The correlation formula is:

$$
T_{l}=\frac{L-M}{R}
$$

Based on the above mathematical derivation, we can get the following dynamic structure diagram of the brushless DC motor after Laplace transform.

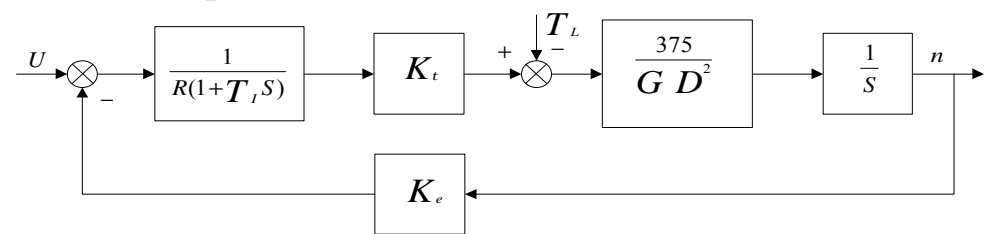

Fig.4- Dynamic structure diagram of the brushless DC motor

Based on the diagram, we can get the transform function of the brushless DC motor:

$$
n(s)=\frac{K_{1}}{T_{2} s^{2}+T_{1} s+K_{2}} U(s)-\frac{1+T_{l} s}{T_{2} s^{2}+T_{1} s+K_{2}} T_{L}(s)
$$

In this equation, $K_{1}=\frac{K_{\tau}}{R}, K_{2}=\frac{K_{\tau} K_{e}}{R} T_{1}=\frac{G D^{2}}{375}{ }^{\prime} T_{2}=T, \frac{G D^{2}}{375}$.

\section{Analysis and Simulation of differential steering control scheme of the robot}

Controlling the robot inspect along magnetic track is actually controlling the speed of two drive motor. The speed control system of the motor can be classified into two categories, the open loop control and the closed loop control. The open loop control responses slowly and can't meet the need of the system. There are several different control methods in the closed loop control, such as PID control, self anti-interference control and sliding mode variable structure control. Among them the PID control has the longest history and wide range of application. Thus, we choose PID control method to apply into robot's control system.

\subsection{Speed control of the brushless DC motor}

We can obtain steady, accurate and fast control effect by adopting double closed loop speed control in the control strategy of the brushless DC motor. The output of the controlled object in the closed-loop control system in turn affects the output of the controller and forms closed loop feedback. The structure of double closed loop control of the brushless DC motor is shown in Fig.5. 


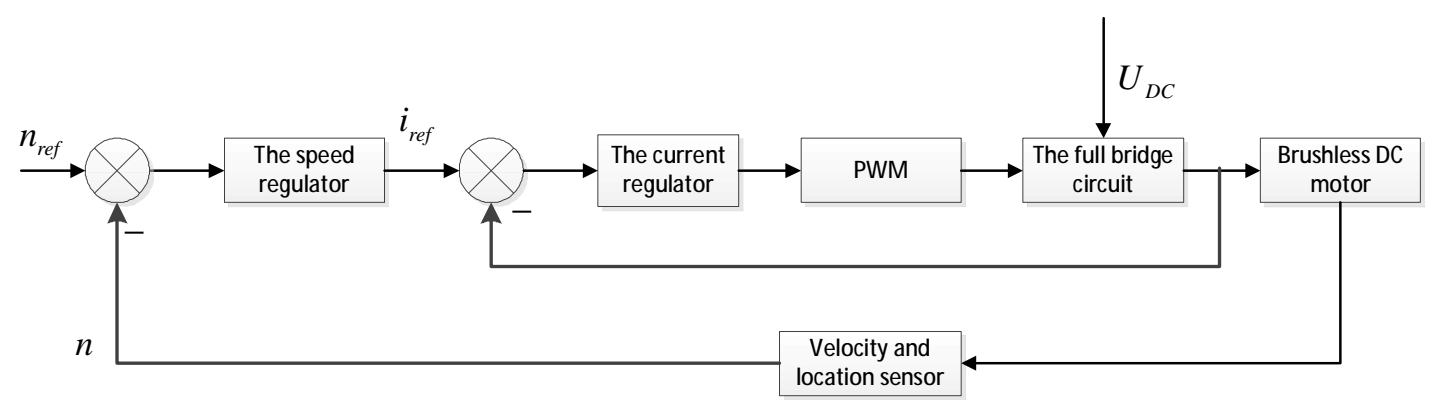

Fig.5- The structure of double closed loop control of the brushless DC motor

In fig. $5, n_{r e f}$ stands for the given speed; $n$ stands for the feedback speed; $i_{\text {ref }}$ stands for the output current of the speed regulator. The control system adopts double closed loop speed control system. The outer loop is the speed regulator and it is the dominant of the control system, it can also make the output change based on the input fast. The inner loop is the current regulator loop and it can accelerate system's dynamic response in the motor speed control process. Under normal circumstances, the dynamic performance of the system is satisfactory.

\subsection{PID control and modeling of the brushless DC motor}

The structure diagram of PID controller is shown in Fig.6.

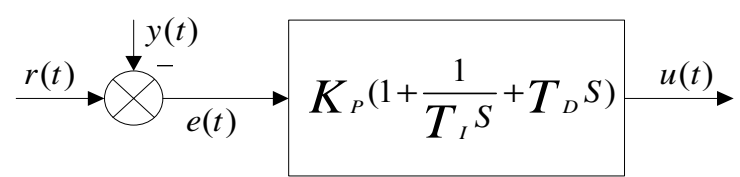

Fig.6- The structure diagram of PID controller

In Fig.6, $r(t)$ stands for the input signal of controller; $e(t)$ stands for the deviation of the input signal; $u(t)$ stands for the output signal of the controller; $y(t)$ stands for the feedback signal; $K_{P}$ stands for the ratio of amplification factor; $T_{I}$ stands for adjustable integration time constant; $T_{D}$ stands for adjustable differential time constant. Thus, the mathematical model of the PID controller can be expressed by the following equation:

$$
u(t)=K_{P}\left[e(t)+\frac{1}{T_{I}} \int_{0}^{t} e(t) d_{t}+T_{D} \frac{d_{e(t)}}{d_{t}}\right]
$$

According to Fig.6 we can know that, the corresponding transfer function is:

$$
G(s)=K_{P}\left(1+\frac{1}{T_{I} s}+T_{D} s\right)
$$

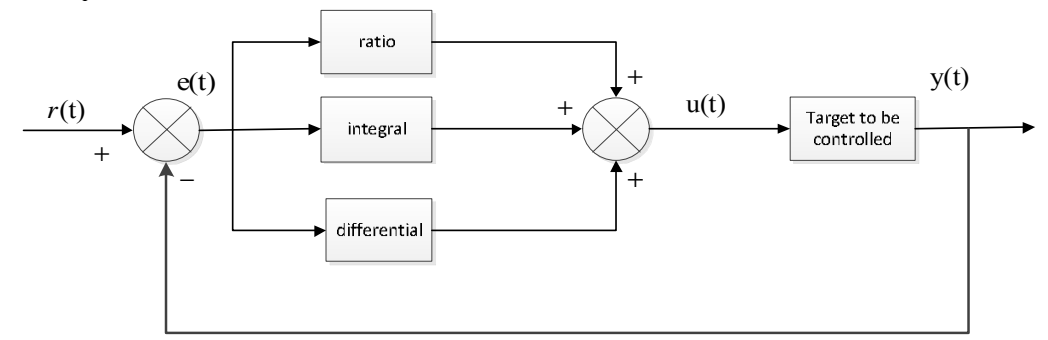

Fig.7- The typical structure diagram of PID control system

Compared to other algorithm, PID controller has the following advantages: it is easy, parameter adjustment is easy and it doesn't need the precise mathematical model of the controlled system. PID controller is widely used in industrial control system. The typical structure diagram of PID control system is shown in Fig.7.

Proportional integral control is usually used in the motor control system. Embedded the PI controller into the brushless DC motor double closed loop speed regulation system and we can get the structure diagram of the control system which is shown in Fig.8. We get the mathematical simulation of the control system by using Matlab / Simulink simulation software and validate the system performance of the brushless DC motor embedded with PID controller. Because of there is no 
simulation model of the brushless DC motor in the library of SIMULINK, we have to establish simulation model by ourselves according to the electrical characteristics of Brushless DC motor; we also have to establish the corresponding double closed loop control system. The structure of corresponding simulation model is shown in Fig.9.

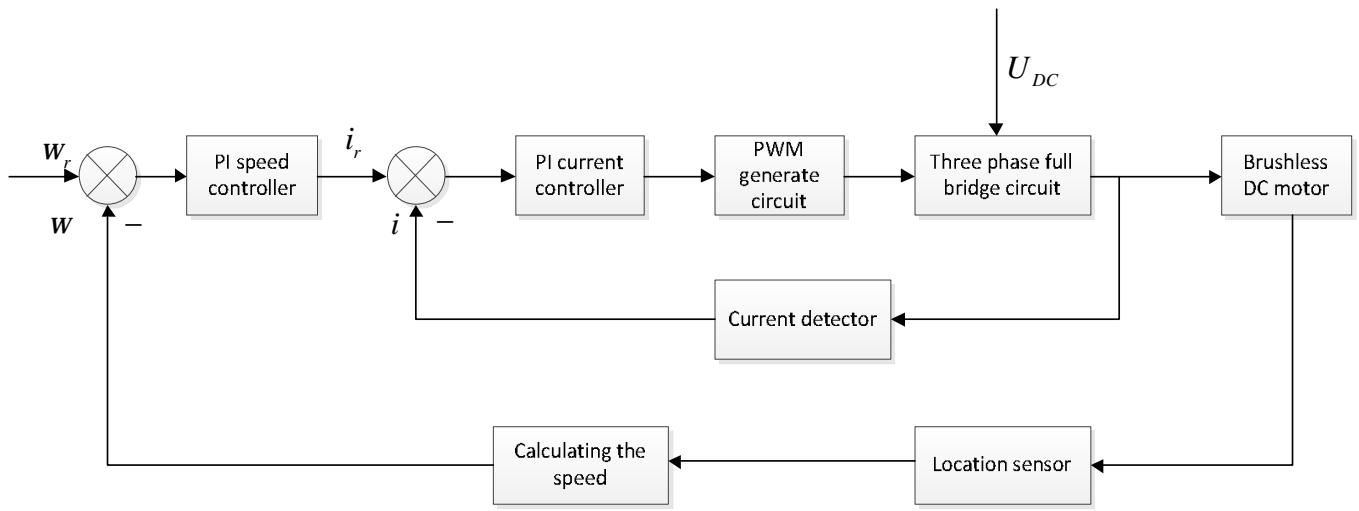

Fig.8- Structure diagram of PID control system in the brushless DC motor
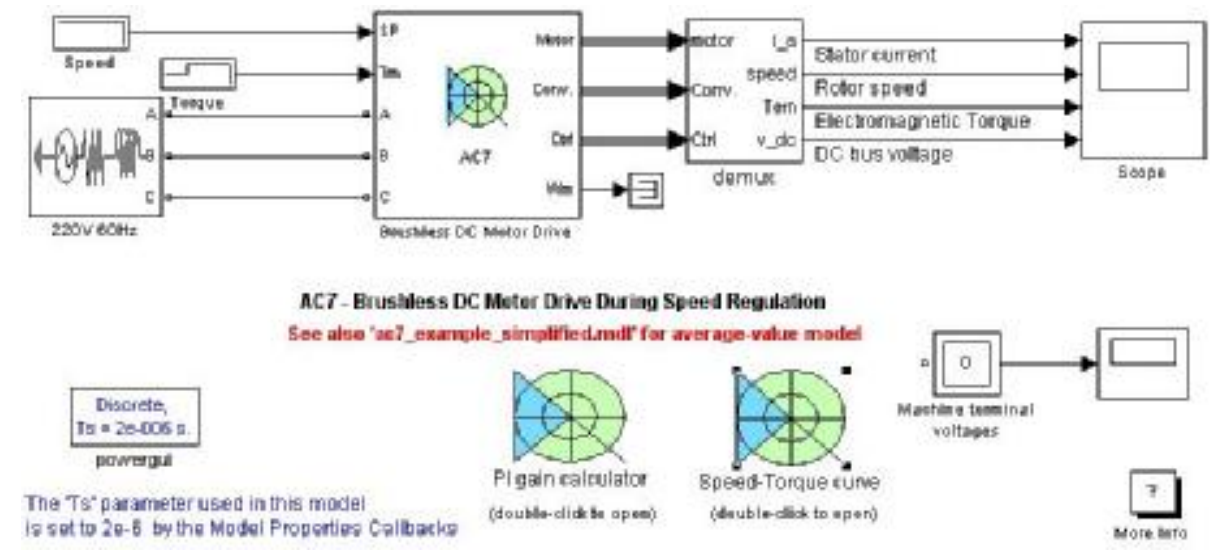

Fig.9- Simulation structure of double closed loop in brushless DC motor

When we are doing the simulation, we set the corresponding parameters according to the parameters on the nameplate of brushless DC motor FBL-909H20301RGN20. The inductance of the stator is $8.5 \mathrm{mH}$, the resistance is $0.2 \Omega$, the rotor flux is $0.175 \mathrm{~Wb}$, the moment of inertia $J$ is $0.089 \mathrm{~kg} \bullet \mathrm{m}^{2}$, and the phase of back electromotive force is $120^{\circ}$. The time of simulating is $2 \mathrm{~s}$, the giving speed is $300 \mathrm{r} / \mathrm{min}$, the initial load torque is $0 \mathrm{~N} . \mathrm{m}$, after $1 \mathrm{~s}$ the load torque is $5 \mathrm{~N} . \mathrm{m}$, the load torque is $10 N . m$ at $1.5 \mathrm{~s}$. We observe the change of waveforms representing speed and torque of the motor in the process of simulation. Fig. 10 is part of waveforms of the speed. Fig. 11 is waveform of motor's torque.

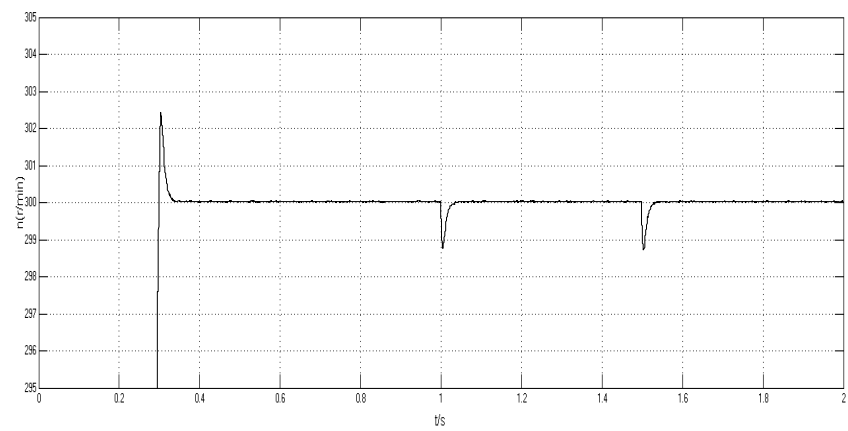

Fig.10- Part of waveforms of the speed

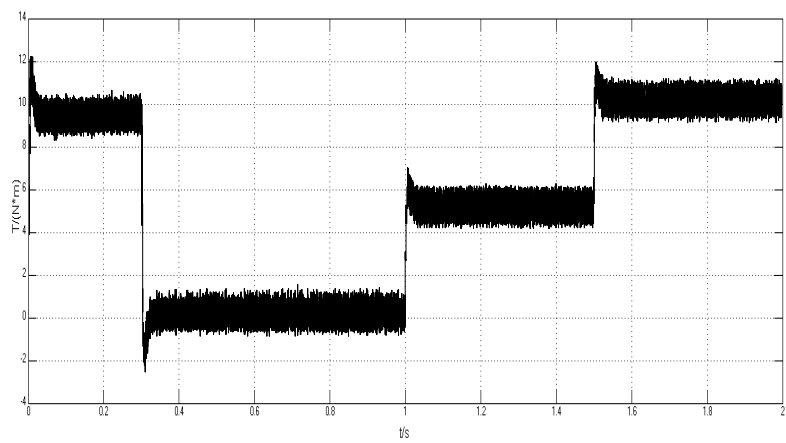

Fig.11- Waveform of motor's torque

According to the waveform of motor's speed and torque, we can find out that when the brushless DC motor starts at no-load state, the response of speed has a large. But the speed rises fast and the 
system tends to be stable quickly. There is almost no pulsation phenomenon after the speed of the motor is stable and the system tends to be stable. When there is a mutation in the motor torque, the system will produce a large overshoot, the response speed of the system is fast; but after the speed of motor is stable, the torque has a certain amount of pulsation. The performance index is shown in table.1.

Table.1-Simulating speed performance of the brushless DC motor

\begin{tabular}{c|c|c|c}
\hline Performance index & $0 \mathrm{~N} . \mathrm{m}$ & $5 \mathrm{~N} . \mathrm{m}$ & $10 \mathrm{~N} . \mathrm{m}$ \\
\hline Overshoot $\sigma \%$ & $0.8 \%$ & $0.5 \%$ & $0.5 \%$ \\
\hline The peak time $\boldsymbol{t}_{P}$ & $0.2 \mathrm{~S}$ & $0.05 \mathrm{~S}$ & $0.02 \mathrm{~S}$ \\
\hline The regulate time $\boldsymbol{t}_{S}$ & $0.1 \mathrm{~S}$ & $0.04 \mathrm{~S}$ & $0.03 \mathrm{~S}$ \\
\hline
\end{tabular}

\section{Conclusion}

We establish the mathematical model of the corresponding motion state by analyzing the motion control principle of the inspection robot. We deduce the mathematical relationship between robot motion direction and speed of the left and right wheel through the analysis of mathematical model. We also establish the robot drive motor, or we can say the mathematical model of the brushless DC motor, and we simulate and analysis the double close loop based control system of the motor through Matlab / Simulink simulation software. From the analysis of simulation we can know that our double close loop control system has a strong tracking ability and a strong anti-interference ability despite it has a high overshoot. It can completely meet the performance requirements and can be used as a substation inspection robot.

The project mentioned in this article is supported by the open fund of key Laboratory of intelligent technology and special robot in Changzhou. (Project number: CZSR2014001)

\section{References}

[1] Zhu Shizhong,Shi Feng, Wang Kun,Sun Weihong,Niu Junpeng and Wang Jianling,research and application of intelligent substation inspection system[J].Electric Power Information Technology,2011,11:92-95.

[2] Li Yujuan,based STM32 substation inspection robot motion control system design and implementation [D].Southwest Jiaotong University,2013.

[3] Guo Lei.Outdoor the study of the magnetic navigation system of autonomous wheeled robot [D].Chongqing University,2012.

[4] Li Yuan. Outdoor autonomous mobile robot navigation system research and design [D].Ocean University of China,2007.

[5]Schulze L,Zhao L.Worldwide development and application of automated guided vehicle systems[J].International Journal of Services Operations and Informatics, 2007,2(2):164-176.

[6] Sun Guokai, Han Gang.Mode of substation inspection comparison and the research and design of intelligent inspection robot[J].The age of electricity 2008:92-94.

[7] Yang Fan.Multiple mobile robots formation control and coordination of transport research

[D].East China University of Science and Technology,2011.

[8] Shen Weiwei.DSP-based wheeled mobile robot control system design[D].Nanjing University of Science and Technology,2010.

[9] Read me for Real View MDk[S].pdf 